\title{
Cutaneous cryptococcal infection presenting as vulvar lesion
}

\author{
KATHLEEN S BLOCHER, * JULIA A WEEKS, $†$ ROBERT C NOBLE*
}

From the *Division of Infectious Diseases, Department of Medicine, and the $†$ Department of Pathology, University of Kentucky College of Medicine, Lexington, Kentucky, United States of America

SUMMARY A woman aged 60, who had been immunosuppressed since receiving a renal transplant 17 years before, developed a painless ulcerated lesion on her right labia majora. Cryptococcus neoformans was demonstrated by culture and biopsy of the lesion. This is the first reported case of a genital skin lesion caused by $C$ neoformans.

The differential diagnosis of a painless genital ulcer typically includes syphilis, granuloma inguinale, and carcinoma. Immunosuppressed patients, however, are subject to several uncommon pathogens, one of which is Cryptococcus neoformans. The lung is probably the primary portal of entry of the cryptococcus, and the organism is apparently carried by the blood stream to the central nervous system and other body sites including the skin. ${ }^{12}$ Cawley et al have estimated that cutaneous lesions occur in about $10 \%$ of patients with cryptococcosis. ${ }^{3}$ To our knowledge, a cryptococcal lesion of the external genitalia has not been reported.

\section{Case report}

A woman with a history of end stage renal disease secondary to polycystic kidneys had undergone a cadaveric renal transplant in December 1969. Thereafter she had good renal function and was maintained for many years taking azathioprine $50 \mathrm{mg}$ orally every day and prednisone $25 \mathrm{mg}$ orally every other day. In late July 1986 , when she was 60 , she noted a small painless papule on her vulva that increased in size during the ensuing two months. The lesion ulcerated, and this was accompanied by slight bleeding and a burning sensation. The patient was otherwise well and had no systemic symptoms. Examination showed, on the inner surface of the right labia majora, erythematous ulceration $1 \mathrm{~cm}$ in diameter with raised margins. The base of the ulcer was intact, and there

Address for reprints: Dr Robert C Noble, Division of Infectious Diseases, Department of Medicine, University of Kentucky College of Medicine, Lexington, Kentucky 40536, USA

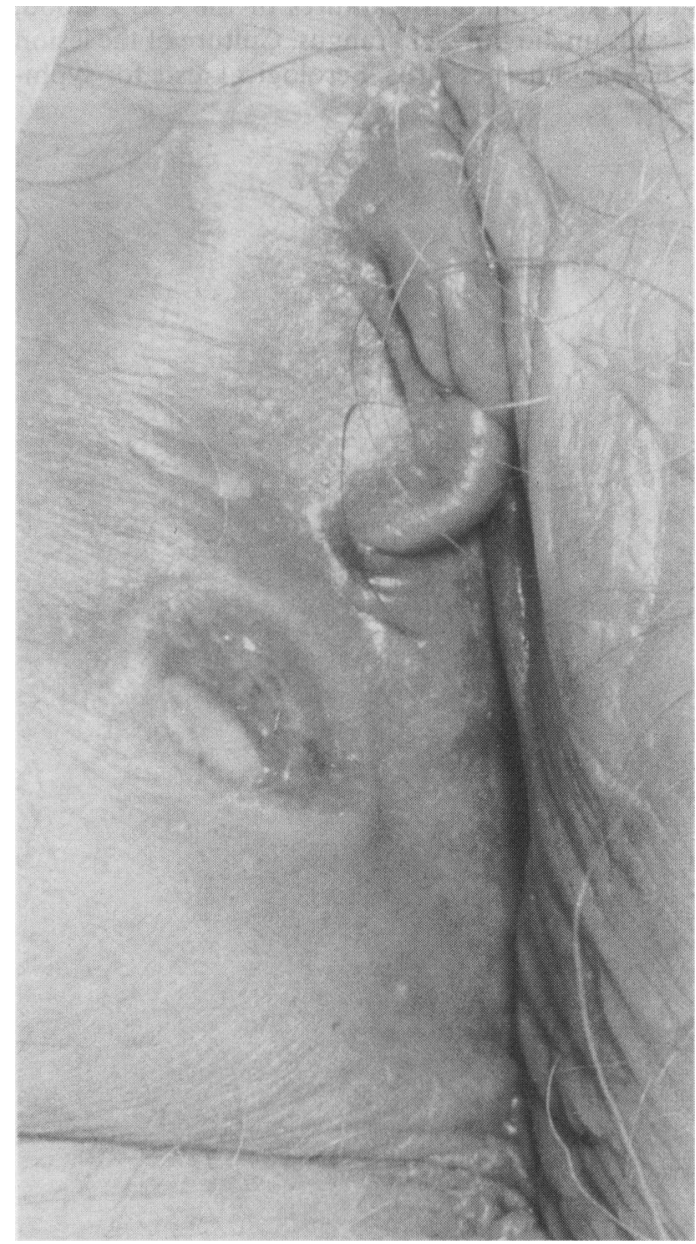

Figure 1 Cryptococcal skin lesion on right vulva eight days after biopsy. 
was no regional adenopathy. Her arms and scalp had numerous keratoacanthomas, actinic keratoses, and seborrhoeic keratoses. No other abnormality was seen.

Abnormal laboratory findings included: white cell count $3.5 \times 10^{9} / 1$ (79\% neutrophils), blood hacmoglobin concentration $101 \mathrm{~g} / \mathrm{l}$, blood urea nitrogen concentration $9.25 \mathrm{mmol} / \mathrm{l}$, serum creatinine concentration $123 \mu \mathrm{mol} / \mathrm{l}$, and serum activities of aspartate aminotransferase $47 \mathrm{IU} / \mathrm{l}$ (normal $=9-36$ ), alanine aminotransferase 47 IU/1 (normal 10-28), and alkaline phosphatase, $137 \mathrm{IU} / \mathrm{l}$ (normal $=45-107)$. The chest radiograph was within normal limits. The cerebrospinal fluid (CSF) had normal glucose and protcin concentrations and cell count and gave negative results to Gram stain, acid fast smear, India ink preparation, and cryptococcal antigen tests. The cryptococcal antigen titre of the scrum was $1 / 8$ as assessed by latex agglutination. Cultures of the CSF, blood, and sputum did not yield fungus. Culture of the lesion did not yield herpes virus. Serological tests for syphi-

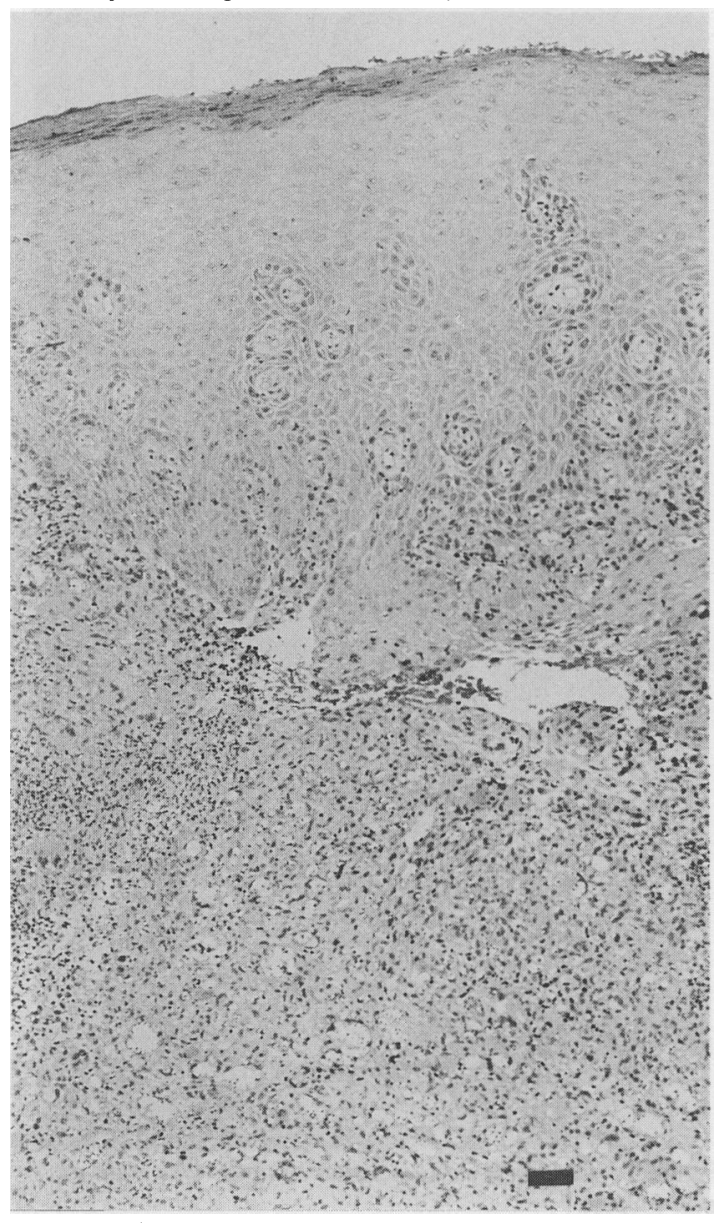

lis were not performed until a later outpatient visit.

The lesion was believed to be carcinoma of the vulva, and in September 1986 a biopsy specimen was taken from the ulcer. Figure 1 shows a photograph of the ulcer eight days after the biopsy. Histopathological examination of the biopsy specimen showed numerous small budding yeasts scattered individually and in gelatinous clusters throughout a diffuse granulomatous inflammatory infiltrate in the dermis (figure 2). Clear zones surrounding the organisms stained bright red with mucicarmine, which is characteristic of mucinous capsules of cryptococcus. Cryptococcus neoformans was grown from a second biopsy specimen. The patient was treated intravenously with amphotericin, and at a follow up outpatient visit in December 1986 the lesion had disappeared. A repeat cryptococcal antigen test of her serum gave a negative result. Serological tests for syphilis gave negative results to the rapid plasma reagin (RPR) card test, the Venereal Disease Research Laboratory (VDRL) slide test, and the

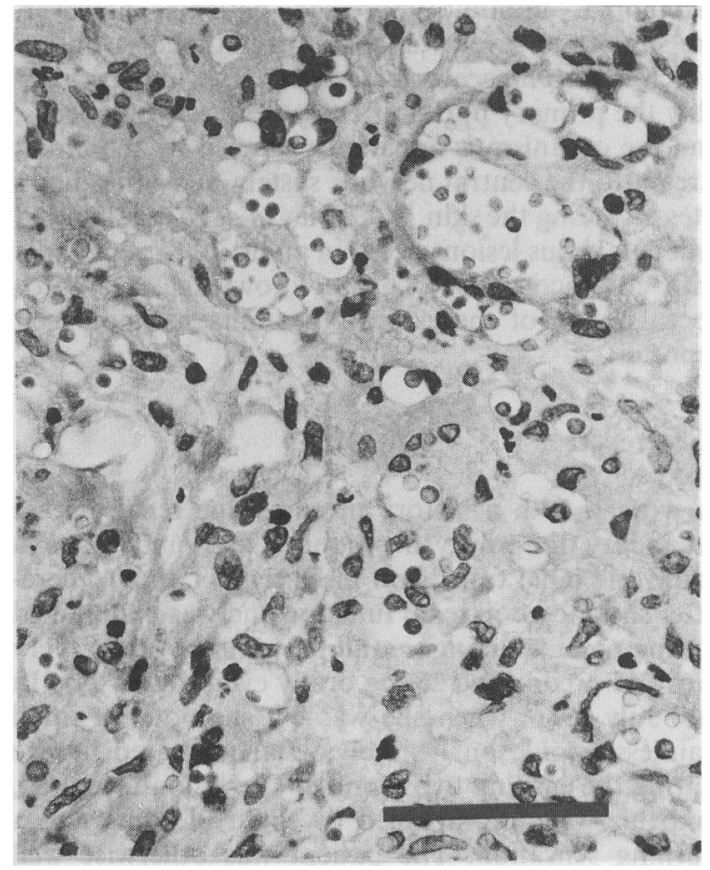

Figure 2 Low power photomicrograph (left) showing epidermal hyperplasia over diffuse granulomatous dermal infiltrate with microabscesses left and centre. Higher magnification of dermal infiltrate (right) showing numerous small yeasts surrounded by clear haloes that correspond to mucinous capsules characteristic of cryptococcus ( haematoxylin and eosin, bars represent $50 \mu \mathrm{m}$ ). 
fluorescent treponemal antibody absorption (FTAABS) test and a positive result to the Treponema pallidum haemagglutination assay (TPHA) for syphilis. The FTA-ABS test and TPHA were repeated without a change in results. The biopsy specimen was microscopically examined for spirochaetes by the Steiner and Warthin-Starry stains, and none were found. The patient had no history of syphilis or any prior positive serological test results for this disease. There was no antibody to the human immunodeficiency virus by enzyme linked immunoabsorbent assay.

\section{Discussion}

Though cryptococcosis may afflict healthy people, it is more common in immunosuppressed patients, including patients with diseases of the reticuloendothelial system, such as Hodgkin's disease, leukaemia, lymphoma, and multiple myeloma. ${ }^{124}$ People with the acquired immune deficiency syndrome are the most recently reported population with an increased risk of cryptococcosis. ${ }^{5}$ Other high risk groups include patients with systemic lupus erythematosis, organ transplant recipients, and other patients who receive therapeutic doses of corticosteroids. ${ }^{12}$ Cryptococcal skin lesions appear to have a predilection for the face, scalp, and neck. Crounse and Lerner reviewed 42 patients and found that $23 \mathrm{had}$ cutaneous lesions of the head and neck; the trunk and arms were more often affected than the legs in the remaining 19.6 More recent experience with patients receiving immunosuppressive drugs, however, has shown several patients with cryptococcal cellulitis that more often affected the legs. ${ }^{27}$ We were unable to find reports of cryptococcal skin lesions of the genitalia.
The morphological variability of cutaneous cryptococcal lesions may preclude an accurate clinical diagnosis. Some of the manifestations of cryptococcal skin infection include papules, nodules, plaques, acneform lesions, non-healing ulcers, superficial granulomas, gummatous lesions, subcutaneous abscesses, ecchymosis, cellulitis, tumour like swellings with and without sinus formation, and lesions resembling molluscum contagiosum. ${ }^{3578}$ Lesions may be solitary or multiple, and painless or painful. The morphological diagnosis of genital ulceration is notoriously difficult, and especially so in an immunodeficient patient. In either normal or immunosuppressed patients, biopsy and culture of a genital lesion may be the quickest route to a definitive diagnosis.

\section{References}

1 Perfect JR, Durack DT, Gallis HA. Cryptococcemia. Medicine (Baltimore) 1983;62:98-109.

2 Schupbach CW, Wheeler CE, Briggaman RA, Warner NA, Kanof EP. Cutaneous manifestations of disseminated cryptococcosis. Arch Dermatol 1976;112:1734-40.

3 Cawley EP, Grekin RH, Curtis AC. Torulosis: a review of the cutaneous and adjoining mucous membrane manifestations. $J$ Invest Dermatol 1950;14:327-41.

4 Zimmerman LE, Rappaport H. Occurrence of cryptococcosis in patients with malignant disease of reticuloendothelial system. Am J Clin Pathol 1954;24:1050-72.

5 Rico MJ, Penneys NS. Cutaneous cryptococcosis resembling molluscum contagiosum in a patient with AIDS. Arch Dermatol 1985;121:901-2.

6 Crounse RG, Lerner AB. Cryptococcosis. Case with unusual skin lesions and favorable response to amphotericin B therapy. Arch Dermatol 1958;77:210-5.

7 Jenning HS, Bradsher RW, McGee ZA, Johnson HK, Alford RH. Acute cryptococcal cellulitis in renal transplant recipients. South Med J 1981;74:1150-3.

8 Rook A, Woods B. Cutaneous cryptococcosis. Br J Dermatol 1962;74:43-9. 